\title{
ESTRUCTURA DE TRES COMUNIDADES DE CALOPTERYX (ODONATA: CALOPTERYGIDAE) CON DIFERENTE COMPOSICIÓN ESPECIFICA
}

\author{
A. Cordero \\ Área de Ecología, Facultad de Biología, Universidad de Santiago de Compostela. 15071 Santiago de Compostela. \\ Spain.
}

Palabras clave: Calopteryx, odonata, spatial distribution, survival, population size.

\begin{abstract}
STRUCTURE OF THREE CALOPTERYX (ODONATA: CALOPTERYGIDAE) COMMUNITIES WITH DIFFERENT SPECIFIC COMPOSITION

Three communities of Calopteryx have been studied from 1985 to 1987 at Galicia (NW of Spain) by markingrecapture techniques. A total of 1275 adults of three species (haemorrhoidalis, virgo and xanthostoma) were marked. The proportion of recaptures was $34 \%$ (1985) and $48 \%$ (1986) in haemorrhoidalis, $54 \%$ (1986) in virgo and $38 \%$ (1987)in xanthostoma. In the study area, the three species are dominant in different environments: haemorrhoidalis in coastal streams, xanthostoma in slow and sunny waters and virgo in rapid waters far from coast. The study was focalized on mobility of adults along the stream and the estimation of population parameters. The results have indicated a mean displacement among recaptures of $15-20 \mathrm{~m}$ for all species, and a significantly higher tendency to fly upstream in inmature than in mature males of haemorrhoidalis. During the maturation period ( 9 days), haemorrhoidalis remains in the stream while virgo and xanthostoma disperse. Mature males concentrate in the water, with a density of 0.4-0.6 males/meter of stream in the middle of July, when the maximum population numbers occur. The maximum observed longevity was 43 days for haemorrhoidalis and 36 days for virgo, with a daily survival rate of about 0.9 , and a mean life span of 10-16 days. Approximately equal numbers of males and females were marked, but the sex ratio became male biased, because females fly away from the stream, and return only for mating and oviposition.
\end{abstract}

\section{INTRODUCCIÓN}

En el género Calopteryx Leach se incluyen los zigópteros de mayor tamaño de Europa. Se trata de especies habitantes de las aguas lóticas, caracterizadas por su coloración metálica corporal y alas pigmentadas, más intensamente en los machos. En España se pueden hallar 3 especies, que a menudo coexisten: haemorrhoidalis, virgo y xanthostoma, cuyos machos se diferencian fácilmente por su coloración alar (castaño en haemorrhoidalis; marrón con reflejos azules en toda

Limnética, 5: 83-91 (1989)

(C) Asociación Española de Limnología, Madrid, Spain la superficie alar en virgo y sólo en su mitad en xanthostoma).

En la reciente revisión de Maibach (1985, 1986, 1987) se concluye que C. xanthostoma debe denominarse C. splendens xanthostoma, por tratarse de una semiespecie (forma altamente diferenciada, pero con aislamiento reproductivo incompleto) de C. splendens, basándose en análisis bioquímicos y en el estudio de la coloración alar. Aquí se hablará de C. xanthostoma, ya que es la única forma del grupo splendens presente en España.

El comportamiento territorial y reproductor de los calopterígidos de Europa occidental ha sido 
ampliamente estudiado por HEYMER (1972,1973). No existe ningún estudio poblacional de este género en la Península Ibérica. En este trabajo se estudia la estructura de tres comunidades, con el fin de conocer los principales parámetros que regulan las poblaciones de Calopteryx (movilidad, probabilidad de supervivencia, longevidad, período de maduración y proporción de sexos).

\section{MATERIAL Y MÉTODOS}

Desde 1985 a 1987 se han estudiado tres comunidades con distinta composición específica dentro del género Calopteryx, mediante marcaje y recaptura de los adultos. Para los marcajes se realizaron números en las alas, utilizando rotuladores de tinta permanente. Para cada observación se anotó el número, sexo, estado de maduración y lugar de recaptura dentro del área de muestreo.

\section{Poblaciones estudiadas}

En 1985 se seleccionó un pequeño canal de $60 \mathrm{~cm}$ de ancho, con prolongada sequía estival, situado en Salcedo (Pontevedra). La zona inspeccionada incluía unos $100 \mathrm{~m}$ del tramo final del canal, donde transcurre entre prados y zonas de matorral. Se trata de una comunidad casi monoespecífica de $C$. haemorrhoidalis (sólo se observaron 2 individuos de C. virgo y, en 1984, 2 de $C$. xanthostoma). Las observaciones tuvieron lugar entre el 19 de junio y el 14 de septiembre (52 muestreos, diariamente del 9 de julio al 20 de agosto). Otras especies de odonatos presentes fueron Cordulegaster boltoni y Lestes viridis.

En 1986 se muestreó un arroyo situado a unos $1200 \mathrm{~m}$ del anterior, de aproximadamente $1,5 \mathrm{~m}$ de ancho, sin sequía estival y a $500 \mathrm{~m}$ de la costa. Se inspeccionó un tramo de unos $300 \mathrm{~m}$, un canal y un manantial cercano. Comunidad compuesta por de $C$. haemorrhoidalis (dominante) y $C$. virgo meridionalis. Las observaciones se realizaron entre el 3 de julio y el 15 de agosto (27 muestreos, diariamente del 5 al 16 de julio). Otras especies presentes: Cordulegaster boltoni, Onychogomphus uncatus, Boyeria irene, Orthetrum coerulescens y Pyrrhosoma nymphula.
En 1987 el estudio se realizó en la orilla del río Umia, a su paso por Portas (Pontevedra). Se eligió un tramo de $90 \mathrm{~m}$, con densa vegetación herbácea en las orillas. En este punto el cauce es poco profundo (menos de 1,5 m) y de $10-12 \mathrm{~m}$ de anchura. La comunidad estaba compuesta por C. xanthostoma (dominante), C. virgo y ejemplares ocasionales de C. haemorrhoidalis. El muestreo se inició el 25 de julio y se interrumpió el 2 de agosto, debido al acusado descenso poblacional, por hallarse $C$. xanthostoma al final de su período de vuelo en esta localidad. Otras especies presentes eran Onychogomphus uncatus, Gomphus simillinus, Oxygastra curtisi, Orthetrum coerulescens, Platycnemis latipes, P. acutipennis y, ocasionalmente, Lestes viridis, Cercion lindeni, Ischnura graellsi y Ceriagrion tenellum.

\section{Análisis de los datos}

Se han utilizado los métodos de Jolly y ManlyParr (Southwood, 1978; BEgon, 1979), que permiten obtener estimas del tamaño de población, proporción de población marcada y probabilidad de supervivencia. Teniendo en cuenta que no se trata de poblaciones aisladas, sino de tramos dentro de cursos de longitud muy superior, las estimas poblacionales se ven afectadas por el error debido al desplazamiento de los individuos fuera del área de muestreo. Por ello, más que como estimas absolutas, son útiles para la comparación entre especies dentro del mismo hábitat.

Para el análisis de los movimientos a lo largo del hábitat, eł arroyo estudiado en 1986 fue dividido arbitrariamente en 14 secciones, situadas en el arroyo principal y en un canal de riego derivado. Estos puntos de referencia no se hallaban a una distancia fija, pero el promedio era de 10$15 \mathrm{~m}$. Una división similar en 18 secciones de $5 \mathrm{~m}$ se realizó en la orilla del río Umia estudiada en 1987. Teniendo en cuenta todas las recapturas de cada individuo (en el caso de varias observaciones en un mismo día se consideró la primera) se ha calculado un «índice de movilidad». Se asignó a cada desplazamiento un valor igual al número de puntos de muestreo recorridos, sin tener en cuenta el sentido. El valor para cada individuo se promedia por el número de desplazamientos observados, y esto proporciona la distancia media 
recorrida por cada individuo entre dos recapturas, en función de los puntos de referencia. Este valor se corrige por la distancia entre puntos de muestreo para obtener el desplazamiento en metros.

La probabilidad de supervivencia basada en un intervalo de más de un día, se ha corregido hallando la raíz de índice $\mathrm{n}+1$, siendo $\mathrm{n}$ el número de días del período omitido (GARrison \& HAFERNIK, 1981). Basándose en la estima promediada de la probabilidad de supervivencia diaria, se calculó la longevidad media, hallando el inverso del logaritmo natural de la estima, cambiado de signo (Cook et al., en Garrison \& HAFERNIK, 1981). A lo largo del trabajo, los resultados se presentan como medias y sus errores standard.

\section{RESULTADOS}

\section{Movimientos y territorialidad}

El valor promedio del índice de movilidad, junto con la proporción de desplazamientos realizados en cada sentido del arroyo, se presenta en la tabla 1. Para los datos globales, no se observa una clara tendencia al desplazamiento en ningún sentido, lo cual está probablemente relacionado con el comportamiento territorial. En el caso de C. haemorrhoidalis, la comparación entre las pautas de movimiento de los individuos jóvenes y maduros indica diferencias significativas para los machos $\left(\chi^{2}\right.$ contingencia $\left.=59,408, p<0,01\right)$ pero no para las hembras ( $\chi^{2}$ contingencia $\left.=4,105 \mathrm{~ns}\right)$. Los machos jóvenes tienen mayor tendencia a desplazarse río arriba que los machos maduros, los cuales, debido a la territorialidad permanecen en la misma zona (jóvenes $[\mathrm{N}=146$ desplazamientos]: $52,1 \%$ río arriba; $32,9 \%$ río abajo y $15,1 \%$ sedentarios; maduros $(\mathrm{N}=408): 25,5 \%$ río arriba; $24,0 \%$ río abajo y $50,5 \%$ sedentarios). Las hembras jóvenes presentan también mayor tendencia, aunque no significativa, a desplazarse río arriba que las maduras $(40,7 \%$ frente a $31,3 \% ; \mathrm{N}=81$ y 240 desplazamientos, respectivamente). La falta de observaciones de individuos jóvenes para $\boldsymbol{C}$. virgo y $\mathrm{C}$. xanthostoma impide comprobar si existe este comportamiento. La mayor proporción de individuos de C. xan- thostoma desplazados río abajo se debe probablemente al fuerte viento de cabecera reinante durante el período de estudio. La tendencia a volar río arriba en muchos insectos acuáticos serviría para contrarrestar la deriva producida por la corriente en el estado larvario (MARGALEF, 1981), y en el caso de Calopteryx cornelia es muy acentuada: el $75 \%$ de 43 individuos se desplazaron río arriba y sólo el $7 \%$ río abajo (HıgAsHi \& UEDA, 1982).

El índice de movilidad (tabla 1) es mayor para las hembras que para los machos en las tres especies, pero esta diferencia sólo es significativa para C. virgo (test $t=2,054, p<0,05$ ). La comparación entre $\mathrm{C}$. haemorrhoidalis y $\mathrm{C}$. virgo indica que los machos de la primera especie se desplazan más que los de la segunda $(1,4$ frente a $1,0 ;$ test $\mathrm{t}=2,116, \mathrm{p}<0,05)$, no existiendo diferencias significativasentre las hembras. Los resultados para C. xanthostoma no son comparables debido a que el hábitat fue distinto. Relacionando los valores del índice con la distancia entre puntos de referencia de cada arroyo se obtiene un

Tabla 1.- Movilidad de los adultos de Calopteryx. El índice de movilidad proporciona el desplazamiento promedio realizado entre recapturas sucesivas, como una función del número de puntos de referencia recorridos (véase el texto). Se presenta asimismo la proporción de desplazamiento río arriba $(+)$, río abajo $(\rightarrow$ y residentes $(0)$. N1, número de individuos; N2, número de desplazamientos. El mayor valor del índice para C. xanthostoma se debe a que la distancia entre puntos de referencia era de $5 \mathrm{~m}$, frente a $10-15 \mathrm{~m}$ para las otras dos especies.

Mobility of adult Calopteryx. The mobility index supplies the mean displacement among succesive recaptures, as a function of the number of reference points travelled (see the text). The proportion of displacements upstream $(+)$, downstream $(\rightarrow$ and residents (0) is also presented. N1, number of individuals; N2, number of displacements. The higher valve of the index for C. xanthostoma is due to that distance among reference points was $5 \mathrm{~m}$, instead of $15-15 \mathrm{~m}$ for the other remaining species.

\begin{tabular}{|c|c|c|c|c|c|}
\hline \multirow[b]{2}{*}{ Especie } & \multirow{2}{*}{$\begin{array}{c}\text { Sexo } \\
(N 1, N 2)\end{array}$} & \multirow{2}{*}{$\begin{array}{l}\text { indice } \\
(\hat{x}+S E)\end{array}$} & \multicolumn{3}{|c|}{ Desplazamientos \% } \\
\hline & & & + & $m$ & 0 \\
\hline \multirow[t]{2}{*}{ C. haemorrhoidalis } & $\sigma^{\prime \prime}(238,554)$ & $1,4 \pm 0,08$ & 32,5 & 26,4 & 41,2 \\
\hline & 우 $(188,321)$ & $1,5 \pm 0,09$ & 33,6 & 33,0 & 33,3 \\
\hline \multirow[t]{2}{*}{ C. virgo } & $\sigma^{7}(45,135)$ & $1,0 \pm 0,16$ & 20,7 & 28,1 & 51,1 \\
\hline & $q(23,50)$ & $1,6 \pm 0,28$ & 32,0 & 36,0 & 32,0 \\
\hline \multirow[t]{2}{*}{ C. xanthostoma } & $\sigma(33,61)$ & $3,5 \pm 0,62$ & 31,1 & 37,7 & 31,1 \\
\hline & $q(11,25)$ & $4,3 \pm 0,84$ & 40,0 & 48,0 & 12,0 \\
\hline
\end{tabular}


desplazamiento promedio entre recapturas de 15-20 m para las tres especies.

El macho de Calopteryx es territorial (PAJUNEN, 1966) y defiende un lugar para la puesta, donde sólo permite que ovipositen las hembras que han copulado con él (Heymer, 1973). Sin embargo, a altas densidades, algunos individuos no consiguen un territorio y vagan por distintas zonas del cau-

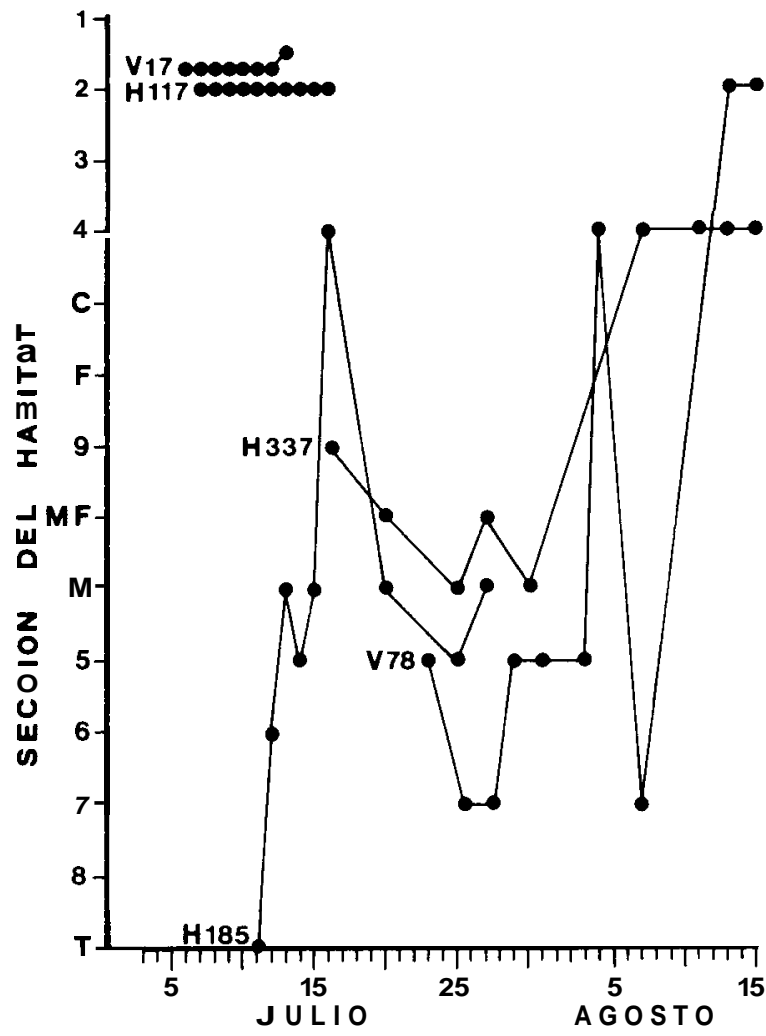

Figura 1.- Pautas de recaptura de los machos territoriales y no territoriales de Calopteryx haernorrhoidalis $(\mathrm{H})$ у $\boldsymbol{C}$. virgo (V). Las 14 secciones del hábitat se presentan ordenadas río abajo desde 1 a $\mathrm{T}$. Los machos territoriales se recapturan en la misma zona ( $\mathrm{H} 117, \mathrm{~V} 17)$, mientras que los no territoriales se recapturan cada día en distinto punto (H185, H337, V78), aunque pueden comportarse como territoriales ocasionalmente.

Recapture patterns of territorial and nonterritorial males of Calopteryx haemorrhoidalis $(\mathrm{H})$ and $\boldsymbol{C}$. virgo (V). The 14 habitat sections are presented downstream from 1 to $\mathrm{T}$. Territorial males are recaptured in the same area (H117, V17), while nonterritorial males are recaptured each day in different section (H185, H337, V78), although they can behave occasionally as territorial.

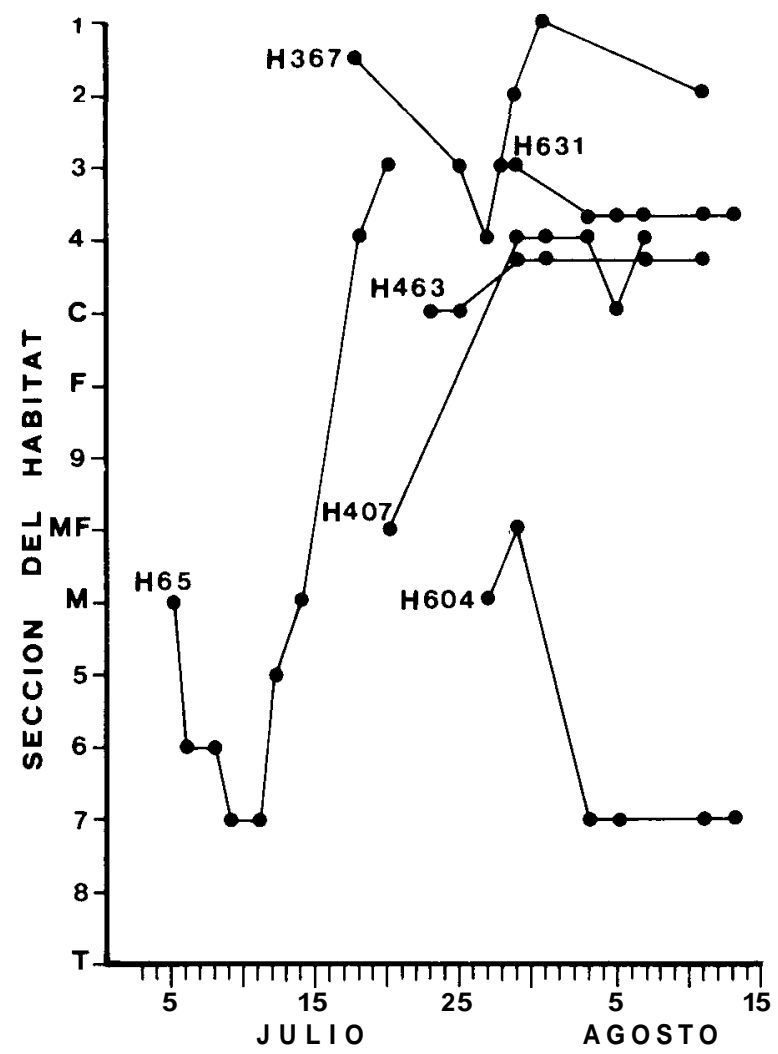

Figura 2.- Pautas de recaptura de 6 hembras de Calopteryx haemorrhoidalis. Algunas hembras (H407, H463, H604, H631) tienden a volver día tras día al mismo punto. Los mismos símbolos que en la figura 1 .

Recapture patterns of 6 females Calopteryx haemorrhoidalis. Some females (H407, H463, H604, H631) return each day to the same area of stream. Symbols are as in figure 1.

ce. En la figura 1 se presentan las pautas de recaptura de los machos, territoriales y no territoriales, de $\boldsymbol{C}$. haemorrhoidalis y $\boldsymbol{C}$. virgo para los que se posee mayor número de observaciones. El ejemplo más claro de macho territorial es el H117, recapturado diariamente entre el 7 y el 16 de julio, siempre en el mismo punto. Un macho no territorial se recaptura sucesivamente en distintos puntos del cauce (H185, H337, V78) y generalmente se trata de individuos que son incapaces de defender un territorio frente a otros machos (HEYMER, 1972). Idénticos resultados se han obtenido para C. xanthostoma.

Aunque las hembras no son territoriales, existe evidencia de que algunos ejemplares tienden a 
volver día tras días al mismo punto (fig. 2), como ocurre con las hembras H407, H463, H604 y H631 de $C$. haemorrhoidalis, para las que se posee mayor número de recapturas. En C. virgo y $C$. xanthostoma existen algunas hembras que siguen esta pauta, pero son necesarios más datos de recaptura. Este comportamiento no parece haber sido señalado para las hembras de ningún Calopteryx.

\section{Período de maduración}

El tiempo promedio hasta el desarrollo de la coloración rojo-carmín abdominal en los machos de C. haemorrhoidalis fue de 9,0 $\pm 2,58$ días $(n=4)$ en 1985 y 8,6 $\pm 0,43(n=24)$ en 1986. El período de maduración es, por tanto, de unos 9 días, lo cual se aproxima a los 10 días estimados por Heymer (1972) en Francia. El color carmín empezó a aparecer no obstante a los $4,8 \pm 1,61$ días $(n=5)$ en 1985 y a los $3,5 \pm 0,57$ días $(\mathrm{n}=10)$ en 1986 .

La pruinescencia abdominal de las hembras se desarrolló en promedio a los 6,8 \pm 0,49 días $(\mathrm{n}=27)$ en 1986 . Ahora bien, como sólo 5 de estas hembras fueron marcadas en el día de su emergencia, y el resto a los 1-2 días, el período de maduración será de unos 9 días, como en los machos. La pruinescencia empieza a aparecer a los 4,8 $\pm 0,78$ días $(\mathrm{n}=12)$ y alcanza un desarrollo máximo a los $21,0 \pm 2,43$ días $(n=6)$.

Estimas semejantes han sido proporcionadas para otros calopterígidos: $10,7 \pm 0,73$ días (intervalo al $95 \%$ ) para los machos de Mnais pruinosa pruinosa (HigAsHI, 1982), 11 días para C. maculata (WAAGE, 1973) y aproximadamente una semana para C. aequabilis, C. amata y C. maculata (NANTEl, 1986). No obstante, Zahner (1960) y BuchHoltz (1951) indican que C. splendens puede madurar en 2 o 3-5 días, respectivamente, lo cual es puesto en duda por HeYMER (1972).

No ha sido posible obtener estimas para C. virgo y $C$. xanthostoma debido a que los individuos jóvenes se alejan del arroyo, al contrario que los de C. haemorrhoidalis. Así, un 52\% (1985) y un $46 \%$ (1986) de los individuos de esta especie fueron marcados antes de la madurez reproductora. Esta proporción fue tan sólo del $5 \%$ en C. virgo y del $12 \%$ en C. xanthostoma, lo que confirma su alejamiento.

\section{Tamaño de población}

En el tramo de arroyo estudiado en 1986, la población de C. haemorrhoidalis estuvo constituida por unos 75-100 machos y 50-75 hembras, observándose claramente un máximo a finales de julio. Con esta población coexistía un pequeño número de individuos de C. virgo, entre 15-20 machos y 10 hembras (fig. 3). Esto indica una densidad máxima de 0,6 machos/m de orilla para $C$. haemorrhoidalis y de 0,07 para C. virgo. En general,
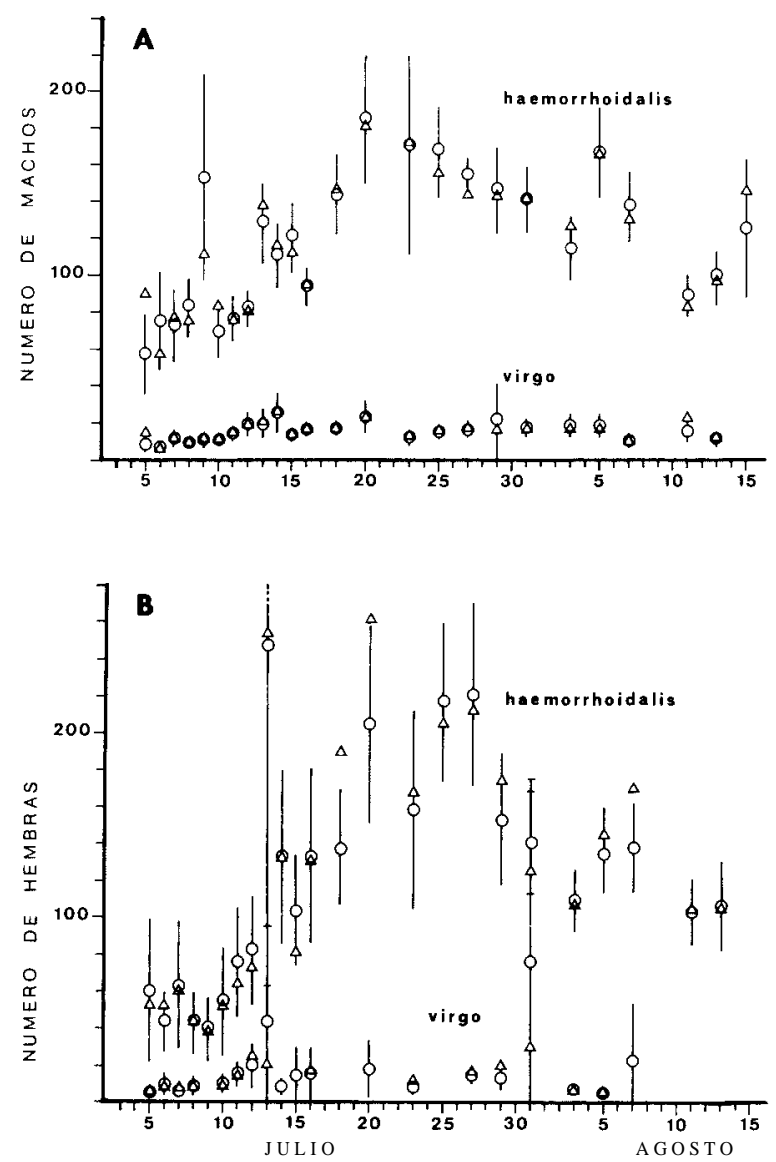

Figura 3.- Estimas del número de individuos para dos poblaciones coexistentes de Calopteryx haemorrhoidalis y $C$. virgo, según los métodos de Jolly (círculos) y Manly-Parr (triángulos). A, machos; B, hembras. Las barras verticales indican \pm SE de las estimas de Jolly.

Estimates of population numbers for two coexisting populations of Calopteryx haemorrhoidalis and $C$. virgo, according to Jolly (circles) and Manly-Parr (triangles) methods. A, males; B, females. Vertical bars are \pm SE of Jolly estimates. 
las estimas fueron más exactas para C. virgo, ya que la intensidad del muestreo fue muy apropiada para su pequeño tamaño poblacional.

Las estimas indican que la población de C. haemorrhoidalis estudiada en 1985 constaba de un número de ejemplares muy semejantes a la de $\mathrm{C}$. virgo en 1986. En el período de estudio, la población de C. xanthostorna del río Umia estaba constituida por pocos individuos: 30-40 machos y 15-20 hembras, coexistiendo con 2-3 de C. virgo, pero su número descendió rápidamente (densidad: $0,4 \mathrm{machos} / \mathrm{m}$ para C. xanthostoma y 0,03 para C. virgo).

En la tabla 2 se presentan los índices de recaptura. Puede observarse que los machos han sido recapturados un mayor número de veces que las hembras en las tres especies (salvo en 1985), y que la proporción de recapturas osciló entre el $34 \%$ de C. haemorrhoidalis en 1985 y el $54 \%$ de C. virgo en 1986.

\section{Longevidad}

Los métodos de Jolly y Manly-Parr permiten obtener estimas de la probabilidad diaria de supervivencia. En la tabla 3 se presentan las estimas promediadas. El método de Manly-Parr proporciona a menudo estimas mayores que 1 , que no tienen sentido. La mayoría de las estimas indican una probabilidad de supervivencia en torno a 0,9 , y una vida media de 10-16 días, lo que, teniendo en cuenta los 9 días del período de maduración, concuerda con los datos de recaptura (tabla 4). Higashi (1976) calculó una probabilidad de 0,943 para Mnais pruinosa, siendo la estima más elevada para un zigóptero la de Ischnura gemina, en donde oscila entre 0,9363 y 0,9579 (GARRISON \& HAFERNIK, 1981).

La longevidad máxima sólo puede obtenerse por intervalo entre marcaje y última recaptura, siendo de 1 a 8 semanas para los zigópteros (CorBET, 1980). Una estima de la vida media puede obtenerse también con este método (tabla 4), pero será una infraestima, ya que el marcaje no coincidirá a menudo con la emergencia, ni la última recaptura con la muerte. La longevidad máxima para C. haernorrhoidalis en Francia es de 39 días (Heymer, 1972), muy semejante a los 43 días hallados en este estudio. La longevidad de
Tabla 3.- Estimas de la probabilidad diaria de supervivencia (S) y longevidad media para Calopteryx.

Estimates of daily survival rate (S) and mean longevity for $\mathrm{Ca}$ lopteryx.

\begin{tabular}{|c|c|c|c|c|}
\hline Especie y ano & Método & $N$ & $S \pm S E$ & $\begin{array}{l}\text { Vida media } \\
\quad \text { (días) }\end{array}$ \\
\hline \multicolumn{5}{|c|}{ C. haemorrhoidalis (1985) } \\
\hline \multirow[t]{2}{*}{ machos } & Jolly & 18 & $0,91 \pm 0,120$ & 10,6 \\
\hline & M-P & 16 & $1.17+0,229$ & - \\
\hline \multirow[t]{2}{*}{ hembras } & Jolly & 14 & $0,92 \pm 0,140$ & 12,0 \\
\hline & M-P & 6 & $1,18 \pm 0,433$ & 一 \\
\hline \multicolumn{5}{|c|}{ C. haemorrhoidalis (1986) } \\
\hline \multirow[t]{2}{*}{ machos } & Jolly & 24 & $0,94 \pm 0,047$ & 16.2 \\
\hline & M-P & 24 & $0,97 \pm 0,052$ & 32,8 \\
\hline \multirow[t]{2}{*}{ hembras } & Jolly & 23 & $0,89 \pm 0,032$ & 8,6 \\
\hline & M-P & 23 & $0.94 \pm 0,028$ & 16,2 \\
\hline \multicolumn{5}{|l|}{ C. virgo (1986) } \\
\hline \multirow[t]{2}{*}{ machos } & Jolly & 23 & $0,86 \pm 0,046$ & 6,6 \\
\hline & M-P & 23 & $1,04 \pm 0,077$ & - \\
\hline \multirow[t]{2}{*}{ hembras } & Jolly & 15 & $0,95 \pm 0,074$ & 19.5 \\
\hline & M-P & 14 & $0,66 \pm 0,128$ & 2,4 \\
\hline \multicolumn{5}{|c|}{ C. xanthostoma (1987) } \\
\hline \multirow[t]{2}{*}{ machos } & Jolly & 6 & $0.66 \pm 0,176$ & 2,4 \\
\hline & M-P & 6 & $0,67 \pm 0,140$ & 2,5 \\
\hline \multirow[t]{2}{*}{ hembras } & Jolly & 4 & $0,89+0,223$ & 8,4 \\
\hline & M-P & 4 & $0.94 \pm 0.250$ & 16.2 \\
\hline
\end{tabular}

Tabla 2.- Índices de recaptura para Calopteryx. Recapture indexes for Calopteryx.

\begin{tabular}{|c|c|c|c|c|}
\hline \multirow[b]{2}{*}{ Especie y año } & \multicolumn{2}{|c|}{$\begin{array}{l}\text { Número de } \\
\text { individuos }\end{array}$} & \multirow{2}{*}{$\begin{array}{c}\% \\
\text { recap- } \\
\text { tura }\end{array}$} & \multirow[b]{2}{*}{$\begin{array}{c}\text { recapturas/ } \\
\text { individuo }\end{array}$} \\
\hline & marcados & $\begin{array}{l}\text { recap- } \\
\text { turados }\end{array}$ & & \\
\hline \multicolumn{5}{|c|}{ C. haemorrhoidalis (1985) } \\
\hline machos & 76 & 22 & 28,95 & 0,83 \\
\hline hembras & 67 & 26 & 38,81 & 0,75 \\
\hline TOTAL & 143 & 48 & 33.57 & 0,79 \\
\hline \multicolumn{5}{|c|}{ C. haemorrhoidalis (1986) } \\
\hline machos & 450 & 238 & 52,89 & 1,24 \\
\hline hembras & 438 & 187 & 42,69 & 0,75 \\
\hline TOTAL & 888 & 425 & 47,86 & 1,00 \\
\hline \multicolumn{5}{|l|}{ C. virgo (1986) } \\
\hline machos & 74 & 45 & 60,81 & 1,80 \\
\hline hembras & 52 & 23 & 44,23 & 0,96 \\
\hline TOTAL & 126 & 68 & 53,97 & 1,45 \\
\hline \multicolumn{5}{|c|}{ C. xanrhosroma (1987) } \\
\hline machos & 88 & 33 & 37,50 & 0,66 \\
\hline hembras & 30 & 11 & 36,67 & 0,80 \\
\hline TOTAL & 118 & 44 & 37,29 & 0,69 \\
\hline
\end{tabular}


Tabla 4.- Longevidad media y máxima para Calopteryx por intervalo entre recapturas. Se presenta las 5 longevidades máximas. No se dispone de datos para C. xanthostoma. Entre paréntesis, vida media excluyendo los individuos no recapturados.

Mean and maximum longevity for Calopteryx, by interval between recaptures. The 5 maximum longevities are presented. No data are available for $C$. xanthostoma. Mean lifespan excluding individuals never recaptured is presented in brackets.

\begin{tabular}{lccc}
\hline Especie y año & $N$ & $X \pm S E$ (días) & Máximo \\
\hline $\begin{array}{l}\text { C. haemorrhoidalis (1985) } \\
\text { machos }\end{array}$ & 76 & $2,7 \pm 0,74(9,4)$ & $40,22,21,20,15$ \\
$\quad$ hembras & 67 & $2.2 \pm 0.58(5,7)$ & $21,20,18,17,12$ \\
$\begin{array}{l}\text { C. haemorrhoidalis (1986) } \\
\quad \text { machos }\end{array}$ & & \\
$\quad$ hembras & 450 & $5,2 \pm 0,37(9,9)$ & $43.43,41,39,36$ \\
$\begin{array}{l}\text { C. vrrgo (19\%) } \\
\text { machos }\end{array}$ & 438 & $3,6 \pm 0.29(8,4)$ & $30,30,29,26,24$ \\
$\quad$ hembras & 74 & $5.3 \pm 0,94(8.7)$ & $36,34,27,26,23$ \\
\hline
\end{tabular}

las hembras es menor, pero esto debe ser el reflejo de su distinto comportamiento, lo que las hace alejarse del agua y por ello ser menos recapturadas (HinNekint, 1987). LABEZKi (1982) halló una longevidad media y máxima para $C$. splendens en Polonia de 20 y 27 días, marcando individuos recién emergidos.

\section{Proporción de sexos}

A pesar de la preponderancia de los machos en los marcajes (y sobre todo en las recapturas), esto se debe a diferencias de comportamiento entre sexos, y la verdadera proporción de sexos sería 1:1 (WaAge, 1980; Hinnekint, 1987). Los resultados del marcaje de Calopteryx están en concordancia con esta explicación (tabla 5). La proporción de sexos es paritaria cuando se consideran sólo los individuos marcados, pero se hace sesgada hacia los machos al considerar los individuos recapturados o el número total de observaciones, ya que los machos se recapturan en mayor proporción y más veces que las hembras.

Es ilustrativo que en la población de C. haernorrhoidalis estudiada en 1985 la proporción de sexos no se desvíe de 1:1 en ningún caso, debido a haber recapturado no sólo en el canal, sino tam- bién en los alrededores. Proporciones de sexos paritarias se dan también en $C$. maculata y $C$. dimidiata (WAAGE, 1980).

\section{DISCUSIÓN}

Los adultos de Calopteryx constituyen importantes poblaciones en las orillas de las aguas lóticas. Los individuos recién emergidos tardan aproximadamente 9 días en alcanzar la madurez sexual, manteniéndose durante esta etapa alejados del agua, salvo en $C$. haemorrhoidalis. En esta especie la mortalidad durante la maduración es semejante a la de la etapa reproductora, ya que se han recapturado el $45 \%$ de los individuos marcados en la primera fase y el $48 \%$ de los marcados en la segunda $\left(\chi^{2}\right.$ contingencia $\left.=0,728, \mathrm{n} \sim\right)$. Entre los depredadores capaces de capturar ejemplares de Calopteryx se encuentran las arañas (en cuyas telas se han hallado los restos de 2 individuos de $C$. haemorrhoidalis, 2 de $C$. virgo y $l$ de C. xanthostoma) y los dípteros asílidos (un macho recién emergido de $C$. haernorrhoidalis capturado por un asílido de la mitad de tamano que su presa).

El estudio de la movilidad revela que los machos de $C$. haemorrhoidalis tienen mayor tendencia a volar río arriba durante la maduración que en la fase reproductora, y que el desplazamiento promedio entre recapturas es de $15-20 \mathrm{~m}$ en las tres especies. La mayor movilidad de los machos de $C$. haemorrhoidalis frente a los de $C$. virgo podría deberse a su mayor densidad, lo que pro-

Tabla 5.- Proporción de sexos (\% de machos) en Calopteryx. Entre paréntesis, tamano muestra ( ${ }^{* *}$ Diferencias significativas respecto a $1: 1$, con $\mathrm{p}<0.01)$.

Sex ratio (percentatge of males) in calopteryx. In brackets, sample size. ( ${ }^{* *}$ Significant differences from $1: 1$, with $\mathrm{p}<0.01$ ).

\begin{tabular}{|c|c|c|c|c|}
\hline \multirow{2}{*}{\multicolumn{2}{|c|}{ Especie y ano }} & \multicolumn{2}{|c|}{ N." individuos $\sigma^{\pi}$} & \multirow{2}{*}{$\begin{array}{c}\text { N." de } \\
\text { observaciones }\end{array}$} \\
\hline & & marcados & recapturados & \\
\hline \multicolumn{5}{|c|}{ haemorrhoidulir } \\
\hline & 1985 & $53.15(143)$ & $45,84(48)$ & $55.758113)$ \\
\hline & 1986 & $50.68(888)$ & $56,00(425)^{* *}$ & $62.95(888)^{* *}$ \\
\hline virgo & 1986 & $58.73(126)$ & $66.18(68)^{* *}$ & $72.68(183)^{* *}$ \\
\hline xanthostoma & 1987 & $74.58(118)^{* *}$ & $75,00(44)^{* *}$ & $70.71(82)^{* *}$ \\
\hline
\end{tabular}


vocaría más interacciones territoriales. Esto está corroborado por el hecho de que las hembras de las dos especies (que no son territoriales) presentan la misma movilidad. Sin embargo también existen persecuciones territoriales entre los machos de distintas especies.

Heymer (1972) ha descrito las pautas de territorialidad de los machos de estas especies, que son similares a las de otros calopterígidos (HIGA. SHI, 1981; Higashi \& Ueda, 1982; NANTEl, 1986; Nomakuchi et al, 1984). Los individuos jóvenes de $C$. haemorrhoidalisforman grupos numerosos, en donde los animales se mantienen mirando al sol y guardando cierta distancia entre ellos (HEYMER, 1972). Esto ocurrió en el punto 7 del arroyo estudiado en 1986.

Teniendo en cuenta que la vida media estimada del adulto maduro es de unos 3-5 días (tabla 4), la actividad reproductora ocupa una pequeña parte de la vida del imago. Como la proporción de sexos es paritaria, los machos que consiguen defender un territorio durante varios días, obtendrán probablemente un gran éxito copulativo. La duración de la copulación es muy corta, lo cual ha impedido realizar muchas observaciones (promedio: $149 \pm 14,4 \mathrm{~s}[\mathrm{~N}=12$, cópula $]$ y $27 \& 7,3$ $\mathrm{s}[\mathrm{N}=7$, transferencia de esperma $]$ para $C$. haemorrhoidalis, y $58 \mathrm{~s}$ [cópula] para sendas parejas de $C$. virgo y $C$. xanthostoma). No obstante, HeYMER (1973), al observar durante 45 minutos a un macho de $C$. virgo, comprobó que copuló con 5 hembras, y la observación de un macho copulando con dos hembras en un breve lapso de tiempo no es rara. Los machos de $C$. maculata pueden retirar hasta el $88 \%$ del esperma almacenado por la hembra de apareamientos previos (WAAGE, 1979). Esto explica el significado adaptativo de la vigilancia sin contacto que los machos de Calopteryx realizan sobre las hembras con las que han copulado, mientras éstas ponen en el interior de su territorio (WAAGE, 1984). Los machos no territoriales pueden copular con las hembras antes de que éstas se acerquen al arroyo, pero serán interceptadas cuando intenten realizar la puesta en el territorio de un macho. En ocasiones las hembras consiguen introducirse en un territorio y ovipositar sin haver copulado con el macho residente (Heymer, 1973), lo que permite el éxito de los machos no territoriales. La comprobación de que las hembras de C. haemorrhoidalistienen tenden- cia a volver al mismo territorio para la puesta en sucesivos días, puede explicar por qué algunas zonas del arroyo, que presumiblemente ofrecen mejores sustratos de puesta, son preferentemente ocupadas por los machos. La densidad en estos puntos puede ser muy elevada, mientras que otras zonas del arroyo (las que permanecen más tiempo sombrías) son raramente ocupadas, produciendo una heterogeneidad en la distribución espacial de los adultos.

Las tres especies de Calopteryx presentes en España poseen una biología bastante similar. Sin embargo, es común que en un río coexistan dos o las tres especies, a pesar de la potencial competencia que debiera producirse. De los muestreos realizados hasta la fecha en diferentes medios lóticos de Galicia, se deduce que $C$. virgo es la especie más abundante, presentándose como especie dominante en todos los ríos y arroyos de agua rápida, alejados de la costa. En el tramo final de los arroyos y canales de riego, la especie mediterránea $C$. haemorrhoidalis sustituye casi totalmente a $C$. virgo, lo que coincide con las observaciones de OCHARÁn (1983) para Asturias. Ésta es también la especie dominante en los canales de riego cercanos a la costa, incluso con prolongada sequía estival, donde ningún otro Calopteryx se desarrolla. Cuando estos medios se secan, en verano, los adultos se dispersan, siendo capaces de localizar otros arroyos, lo que permite su subsistencia en estas condiciones (CORDERO, en preparación). Por último C. xanthostoma es la menos abundante, habiendo sido hallada como especie dominante sólo en aguas lentas y soleadas. No obstante, se presenta en pequeño número en zonas dominadas por $C$. virgo.

La exclusión de otras especies sólo se produce en los canales más pequeños, que soportan poblaciones monoespecíficas. Únicamente un estudio de los diferentes requerimientos de las larvas podría dilucidar esta cuestión, pero esto es, de momento, irrealizable, ya que las larvas (particularmente las jóvenes) son prácticamente indistinguible .

\section{AGRADECIMIENTOS}

Al Dr. A. Carballeira, por sus comentarios a las primeras versiones del manuscrito. 


\section{Bibliografía}

BEGON, M., 1979. Investigating Animal Abundance. Edward Arnold, Londres. 97 pp.

BuCHHOLTz, C., 1951. Untersuchugen an der Libellen-Gattung Calopteryx-Leach unter besonderen Berücksichtigung ethologischer Fragen. Z. Tierpsychol. 8: 273-293.

Corbet, P.S., 1980. Biology of Odonata. Ann. Rev. Entomol. 25: 189-217.

Garrison, R.W. \& J.E. HaferniK, 1981. Population structure of the rare darnselfly, lschnura gemina (Kennedy) (Odonata: Coenagrionidae). Oecologia 48: 377-384.

Heymer, A., 1972. Comportements social et territorial des Calopterygidae (Odon. Zygoptera). Ann. Soc. ent. Fr. (N.S.) 8 (1): 3-53.

Heymer, A., 1973. Etude du comportement reproducteur et analyse des méchanismes déclencheurs innés (MDI) optiques chez les Calopterygidae (Odon. Zygoptera). Ann. Soc. ent. Fr. (N.S.) 9(1); 219-255.

Higashi, K., 1976. Ecological studies on the population of Mnak pruinosa Selys (Odonata: Calopterygidae). I. Population density, survival rate and daily activities in the adult damselfly. Physiol. Ecol. Japan 17(1/2): 109-116 (En japonés).

Higashi, K., 1981. A description of territorial and reproductive behaviours in Mnais pruinsa Selys (Odonata Calopterygidae). J. Fac. lib. Arts Saga Univ. 13: 123-140.

HigASHI, K., 1982. Length of maduration period and daily food consumption of inmature damselfly Mnais pruinosa pruinosa Selys (Zygoptera: Calopterygidae). Tombo 25 (1-4). 23-26.

Higashi, K. \& T. Ueda, 1982. Territoriality and movement pattern in a population of Calopteryx cornelia (Selys) (Zygoptera: Calopterygidae). Odonatologica 11 (2): 129-137.

HiNNEKINT, B.O.N., 1987. Population dynamics of Ischnura e. elegans (Vander Linden) (Insecta: Odonata) with special reference to morphological colour changes, female polymorphisrn, rnultiannual cycles and their influence on behaviour. Hydrobiologia 146: 3-31.

LABEZKI, A., 1982. Researches concerning the dispersion in terrain and the life length of some species of dragonflies (Odonata). Rocz. Akad. Roln. Pozn. 140: 76-90 (En polaco).
Maibach, A., 1985. Révision systématique du genre Calopteryx Leach (Odonata: Zygoptera) pour l'Europe Occidentale. I. Analyses biochimiques. Mitt. schweitz. ent. Ges. 58 : 477-492.

Maibach, A., 1986. Révision systématique du genre Calopteryx Leach (Odonata: Zygoptera) pour l'Europe Occidentale. II. Analyses morphologiques et synthese. Mitt schweitz. ent. Ges. 59: 389-406.

MaibaCh, A., 1987. Révision systématique du genre Calopteryx Leach por I'Europe Occidentale (Zygoptera: Calopterygidae). 3. Révision systématique, étude bibliographique, désignation des types et clé de détermination. Odonatologica 16(2): 145-174.

Margalef, R., 1981. Ecología. Planeta. Barcelona, 252 pp.

NANTEL, F., 1986. Biologie et comportement territorial de trois espèces de Calopteryx Leach (Odonates: Calopterygides), Comté de Bellechase. Québec. Fabreries 12(2): 25-45.

Nomakuchi, S., K. Higashi, M. Harada \& M. Maeda, 1984. An experimental study of the territoriality in Mnak pruinosa pruinosa Selys (Zygoptera: Calopterygidae). Odonatologica 13(2): 259-267.

OCHARÁN, F.J., 1983. Calopteryx haemorrhoidalis asturica, nueva subespecie de caballito del diablo del norte de España (Odonata: Zygoptera). Bol. Cien. Nat. I.D.E.A. 31: 3-10.

PAJunEn, V.I., 1966. Aggresive behaviour and territoriality in a population of Calopteryx virgo L. (Odon., Calopterygidae). Annls. Zool. Fenn. 3: 201-214.

Southwood, T.R.E., 1978. Ecological methods. Chapman \& Hall., Londres. 524 pp.

WAAGE, J.K., 1973. Reproductive behaviour and its relation to territoriality in Calopteryx maculata (Beauvois) (Odonata: Calopterygidae). Behaviour 47(3-4): 240-256.

WAaGe, J.K., 1979. Dual function of the darnselfly penis: sperm removal and transfer. Science 203: 916-918.

WAAGE, J.K., 1980. Adult sex ratio and fernale reproductive potential in Calopteryx (Zygoptera: Calopterygidae). Odonatologica 9(3): 217-230.

WAaGe, J.K., 1984. Sperm competition and the evolution of odonate matingsystems. In: Sperm competition and the evolution of animal mating systems (R.L. SMITH, ed): 251-290. Academic Press, Orlando.

ZAHNER, R., 1960. Über die Bindung der Mitteleuropaischen Calopteryx-Arten (Odonata: Zygoptera) an den Lebensraurn stromen Wassers. II. Der Anteil des Imagines an der Biotopbindung. Int. Rev. Hydrobiol. 45: 101-123. 
